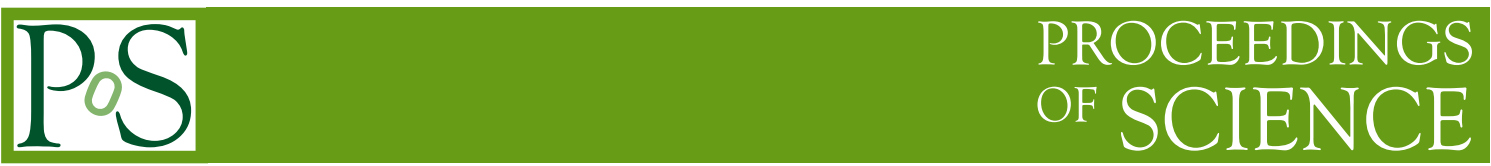

\title{
New neutrino interactions at large colliders
}

\author{
Francisco del Águila*, Juan Antonio Aguilar-Saavedra ${ }^{\dagger}$ \\ Depto. de Fsica Teórica y del Cosmos and CAFPE, U. de Granada, E-18071 Granada, Spain \\ E-mail: faguila@ugr.es, jaas@ugr.es
}

\section{Jorge de Blas}

Department of Physics, University of Notre Dame, Notre Dame, Indiana 46556, USA

E-mail: jdeblasmend.edu

\begin{abstract}
We update present bounds on the helicity of the neutrinos produced in muon decay, including $e^{+} e^{-} \rightarrow \bar{f} f$ LEP 2 data. These significantly reduce the limits derived from all the other electroweak precision data. In Standard Model extensions designed to maximize the RH neutrino production in such a decay the neutrino deficit eventually observable in a near detector at a neutrino factory can be of the order of $5 \%$. Motivated by the current LHC run at $7 \mathrm{TeV}$, we also update previous work providing discovery limits on see-saw mediators at this centre of mass energy. Lepton triplets with $200 \mathrm{GeV}$ could be discovered with luminosities of $1-1.5 \mathrm{fb}^{-1}$. Scalar triplets of the same mass might be seen with $0.75-3 \mathrm{fb}^{-1}$. What makes their search also attractive in the first LHC analyses.
\end{abstract}

35th International Conference of High Energy Physics - ICHEP2010,

July 22-28, 2010

Paris France

\footnotetext{
*Speaker.

$\dagger$ This work has been partially supported by MICINN (FPA2006-05294), Junta de Andalucía (FQM 101, FQM 03048) and the European Union (MRTN-CT-2006-035505).
} 


\section{Introduction}

Neutrino masses and neutrino interactions manifest at two very different energy scales. Thus, while neutrino masses are in the $\mathrm{eV}$ range or below, weak interactions occur near the $\mathrm{TeV}$, at a scale $10^{12}$ times larger. As a consequence, neutrino masses have only showed up at neutrino oscillation experiments where a long baseline enhances their effect [1], but they can be completely neglected in experiments where the relevant energies are of the electroweak order, $v \sim 246 \mathrm{GeV}$, as in large collider experiments. This is the whole story within the minimal Standard Model (SM) extension with tiny Dirac neutrino masses (Yukawa couplings), or with small Majorana neutrino masses from a highly suppressed dimension five Weinberg operator $\left(\mathscr{O}_{5}\right)_{i j}=\overline{\left(l_{L}^{i}\right)^{c}} \tilde{\phi}^{*} \tilde{\phi}^{\dagger} l_{L}^{j}$ (see-saw mechanism) [2]. However, this situation can be totally reversed if new interactions involving light left (LH) and/or right-handed (RH) neutrinos exist near the electroweak scale [3].

In this short communication we revise two interesting cases. The possibility that there are light Dirac or Majorana RH neutrinos with appropriate new weak interactions which may be observable at a large collider and, in particular, at a neutrino factory [4], or on the contrary, that there are no light RH neutrinos but the see-saw mediators of the light Majorana masses for LH neutrinos live near the $\mathrm{TeV}$ at the LHC reach [5].

\subsection{Observable RH neutrino production}

A fair question is how well do we know that light neutrinos are LH ? or to be more concrete, which helicity have the neutrinos produced in muon decay ? [6]. From pion decay into muons we know that the muon charged interactions are mainly LH, and electroweak precision data (EWPD) require this to be so with a precision of few per ten thousand within the SM [1]. This is reflected by the width of the curves in Fig. 1. However, if we allow for light RH neutrinos and new fourfermion interactions of the electroweak size up to a factor of a few, and possible model dependent cancellations, present experimental constraints leave room for light RH neutrinos observable at a neutrino factory, and new mediators observable at LHC [4, 7]. Indeed, although limits on new physics described by dimension six operators are typically of the order of 1-10\% [8], if we allow for cancellations some deviations from the SM can be larger. In Fig. 1 (left) we show two examples (see $[4,9]$ for definitions and details) where the large deviations correspond to the relatively weak bounds (straight lines and crosses) on $\delta g_{L L}^{V}$ and $g_{R R}^{S}$.

It must be noticed, however, that although these SM deviations could be observable at a large $v$ factory as a deficit of the neutrinos detected by a near detector of at most 8.5 (4.5) \% through inverse muon decay (neutrino-nucleon) and an adequate, model independent dimension six operator addition (case E1 in Fig. 1 (right)), such an addition is only designed to maximize this effect while preserving present experimental limits, including those from rare processes. When one also demands that such an addition results from a definite model, the possible deviations are reduced (for instance, to 4 (2) \% in case E2 in Fig. 1 (right)). Finally, it must be stressed that these departures from the SM predictions are compatible with all EWPD, including in particular $e^{+} e^{-} \rightarrow \bar{f} f$ LEP 2 data (right panel in Fig. 1). These appear to be quite restrictive, not only reducing the new parameter space as shown by comparing the left and right panels in Fig. 1, but requiring extra additions to maintain the agreement with experimental data while allowing for relatively large $\delta g_{L L}^{V}$ and $g_{R R}^{S}$ values, for example $\left(\mathscr{O}_{e e}\right)_{e e \mu \mu}=\frac{1}{2}\left(\overline{e_{R}} \gamma_{\alpha} e_{R}\right)\left(\overline{\mu_{R}} \gamma^{\alpha} \mu_{R}\right)$ in the two cases depicted in Fig. 1 (right). 

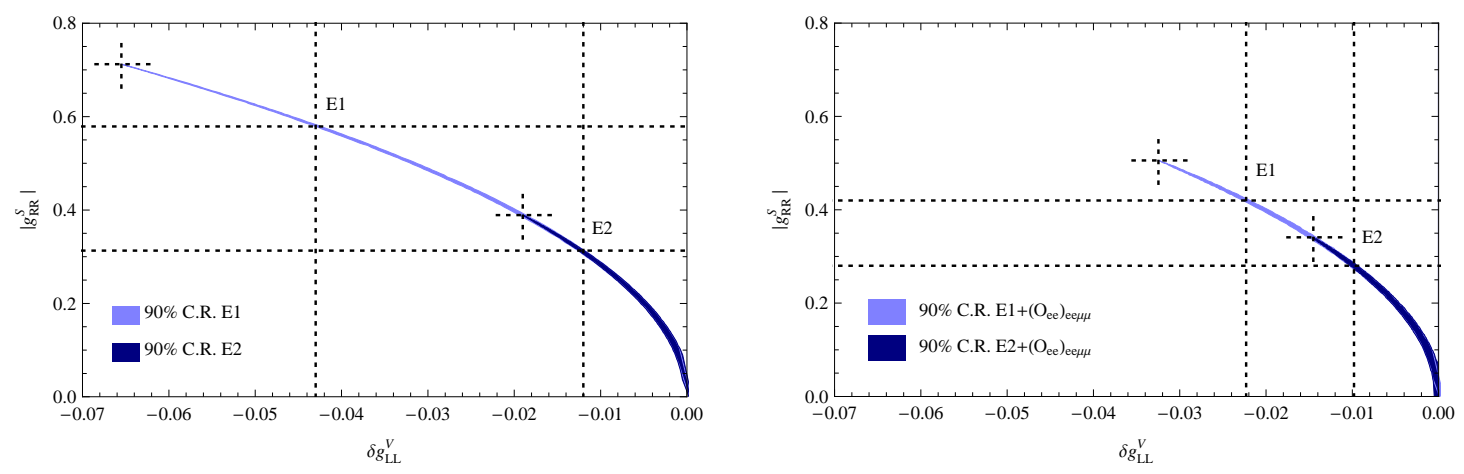

Figure 1: (Left) $90 \%$ C.L. bounds from a global fit using EWPD (without LEP 2 data) to appropriate extra dimension six operators involving light LH (abscisse) and RH (ordinate) neutrinos. E1 and E2 stand for the model independent and the specific model addition described in [4], respectively. (Right) The same including also LEP 2 data in the fit and considering the extra four lepton operator $\left(\mathscr{O}_{e e}\right)_{e e \mu \mu}$. The crosses define the extremes of the corresponding $90 \%$ confidence regions for the global fit to the new parameters.

\subsection{Observable see-saw mediators at LHC}

Light neutrinos may be only LH and get their masses through the Weinberg operator $\left(\mathscr{O}_{5}\right)_{i j}$ after spontaneous symmetry breaking. This dimension five operator results from the tree-level exchange of fermion singlets $N$ and/or triplets $\Sigma$, and/or scalar triplets $\Delta$. Which are known as type I, III and II see-saw mechanism, respectively $[2,3,10]$. In this case if these heavy mediators have masses near the $\mathrm{TeV}$, being then eventually observable at $\mathrm{LHC}$, the smallness of the light neutrino masses requires an extremely small $\sim 10^{-12}$ effective coupling $x_{i j}$ multiplying $\left(\mathscr{O}_{5}\right)_{i j}$. What stands for very small mixing angles in the fermionic case, $\left|V_{\ell N, \ell \Sigma}\right| \sim 10^{-6}$, or a minuscule effective lepton number breaking parameter in the scalar one, $\sim 10^{-12}$. Such small numbers translate into negligible mediator production cross sections at large colliders if their production mechanism is proportional to them, as in the fermion singlet case in the absence of further interactions ${ }^{1}$. (Otherwise, its production rate can be of the electroweak size, as for instance in Left-Right models [3, 10].) In the triplet case their production is through electroweak interactions. In such a case they are observable at $\mathrm{LHC}$ for masses below the $\mathrm{TeV}$. Here we present the corresponding results for a center of mass energy of $7 \mathrm{TeV}$. In Table 1 we gather the luminosity required for a $5 \sigma$ discovery of the see-saw triplet messengers in three different multi-lepton channels. As for a center of mass energy of 14 $\mathrm{TeV}[5,12]$, the four cases considered can be distinguished by comparing the number of events in the different samples. The pre-selection and selection criteria are also similar, with a good reconstruction of the heavy resonance masses, as shown in Fig. 2 for scalar triplets with a mass of $200 \mathrm{GeV}$ and a luminosity of $30 \mathrm{fb}^{-1}$.

\section{References}

[1] K. Nakamura et al. [Particle Data Group], J. Phys. G 37 (2010) 075021.

\footnotetext{
${ }^{1}$ Although the mixing angle $V$ is decoupled from the light neutrino masses, as in the case of quasi-Dirac heavy neutrinos, EWPD require $\left|V_{e N, \mu N, \tau N}\right|<0.05,0.03,0.09$ at $95 \%$ C.L. $[9,10,11]$. What makes difficult to observe them at LHC $[5,12]$.
} 

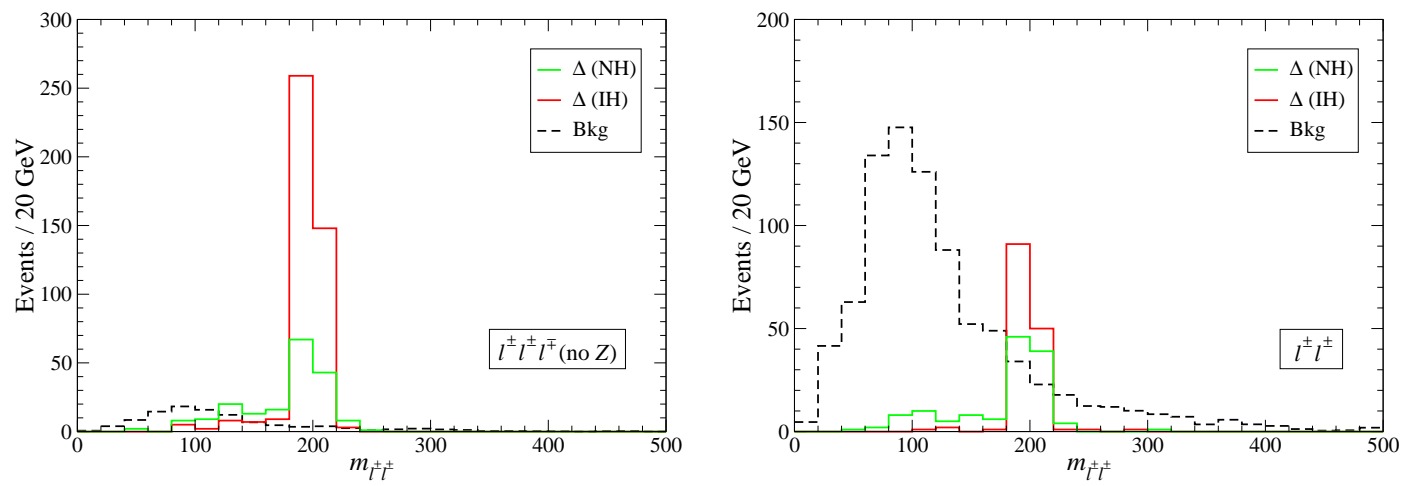

Figure 2: Reconstructed scalar triplet mass in the $\ell^{ \pm} \ell^{ \pm} \ell^{\mp}$ (no $Z$ ) (left) and in the $\ell^{ \pm} \ell^{ \pm}$(right) samples for a center of mass energy of $7 \mathrm{TeV}$ and an integrated luminosity of $30 \mathrm{fb}^{-1}$.

$\begin{array}{cccc} & \ell^{ \pm} \ell^{ \pm} \ell^{\mp}(\text { no } Z) & \ell^{ \pm} \ell^{ \pm}(\text {no } p t \text { ) } & \ell^{+} \ell^{+} \ell^{-} \ell^{-} \\ \Delta(\mathrm{NH}) & 2.7 & 5.9 & 11.5 \\ \Delta(\mathrm{IH}) & 0.74 & 2.1 & 2.0 \\ \Sigma_{\mathrm{M}} & 2.0 & 1.4 & 3.6 \\ \Sigma_{\mathrm{D}} & 0.97 & - & 1.0\end{array}$

Table 1: Luminosities (in $\mathrm{fb}^{-1}$ ) required for $5 \sigma$ discovery at LHC with a center of mas energy of $7 \mathrm{TeV}$ for the models in the left column (scalar triplets $\Delta$ coupling to light neutrinos with a normal $\mathrm{NH}$ or an inverted IH mass hierarchy, and Majorana M or Dirac D fermion triplets $\Sigma$ ) in the final states indicated. A dash stands for an unobservable signal.

[2] R. N. Mohapatra and P. B. Pal, World Sci. Lect. Notes Phys. 60 (1998) 1 [World Sci. Lect. Notes Phys. 72 (2004) 1].

[3] P. Nath et al., Nucl. Phys. Proc. Suppl. 200-202 (2010) 185 [arXiv:1001.2693 [hep-ph]].

[4] F. del Aguila, J. de Blas, R. Szafron, J. Wudka and M. Zralek, Phys. Lett. B 683 (2010) 282 [arXiv:0911.3158 [hep-ph]].

[5] F. del Aguila and J. A. Aguilar-Saavedra, Nucl. Phys. B 813 (2009) 22 [arXiv:0808.2468 [hep-ph]].

[6] P. Langacker and D. London, Phys. Rev. D 39 (1989) 266.

[7] F. del Aguila, J. de Blas, A. Carmona and J. Santiago, Fortsch. Phys. 58 (2010) 675 [arXiv:1003.5799 [hep-ph]].

[8] C. Biggio, M. Blennow and E. Fernandez-Martinez, JHEP 0908 (2009) 090 [arXiv:0907.0097 [hep-ph]].

[9] J. de Blas, Ph.D. Thesis.

[10] F. del Aguila, J. A. Aguilar-Saavedra and J. de Blas, Acta Phys. Polon. B 40 (2009) 2901 [arXiv:0910.2720 [hep-ph]].

[11] F. del Aguila, J. de Blas and M. Perez-Victoria, Phys. Rev. D 78 (2008) 013010 [arXiv:0803.4008 [hep-ph]].

[12] F. del Aguila and J. A. Aguilar-Saavedra, Phys. Lett. B 672 (2009) 158 [arXiv:0809.2096 [hep-ph]]. 